\title{
Phase I dose-escalation study of chiauranib, a novel angiogenic, mitotic, and chronic inflammation inhibitor, in patients with advanced solid tumors
}

\author{
Yongkun Sun ${ }^{1}$, Lin Yang ${ }^{1}$, Xuezhi Hao ${ }^{1}$, Yutao Liu', Jinwen Zhang ${ }^{2}$, Zhiqiang Ning ${ }^{2}$ and Yuankai Shi ${ }^{1 *}$ (D
}

\begin{abstract}
Background: Chiauranib is a novel orally active multi-target inhibitor that simultaneously inhibits the angiogenesisrelated kinases (VEGFR2, VEGFR1, VEGFR3, PDGFRa, and c-Kit), mitosis-related kinase Aurora B, and chronic inflammationrelated kinase CSF-1R. This phase I dose-escalation study was to determine the maximum tolerated dose (MTD), safety, pharmacokinetics, and preliminary antitumor activity of chiauranib in patients with refractory advanced solid tumor and lymphoma.
\end{abstract}

Methods: Eighteen patients were treated with continuous dosing of chiauranib from 10 to $65 \mathrm{mg}$ once daily in a doseescalation $3+3$ design and evaluated in 28-day cycles. Pharmacokinetic profile of plasma chiauranib was analyzed in both single and multiple dose studies.

Results: Dose-limiting toxicity (DLT) as of grade 3 hypertension occurred in two patients at $65 \mathrm{mg} /$ day, and one dose level below as MTD was $50 \mathrm{mg} /$ day. The most common treatment-related adverse events included fatigue (61.1\%), proteinuria (44.4\%), hematuria (38.9\%), hypothyroidism (38.9\%), hypertriglyceridemia (33.3\%), and hypertension (33.3\%). A linear and dose-dependent pharmacokinetic profile of chiauranib was characterized with rapid absorption and slow elimination feature in both single and multiple dose studies. The accumulative exposure of chiauranib reached the steady state within 8 days and was approximately increased by twofold as those in the single dose study. No complete or partial response was observed, and 12 patients (66.7\%) achieved stable disease (SD).

Conclusions: Chiauranib demonstrated an acceptable safety and favorable pharmacokinetic profile with potential antitumor activity. Several phase Ib/II clinical studies are currently under further investigation.

Trial registration: NCT, NCT02122809. Registered 25 April 2014

Keywords: Advanced solid tumors, Chiauranib, Dose-escalation, Pharmacokinetics, Phase I study, Safety

\section{Background}

Tumorigenesis is a multi-step process involving in distinctive and complementary hallmark capabilities that enable tumor growth and metastatic dissemination [1]. Accordingly, the phenotypic and functional heterogeneity of tumor cells especially in tumor microenvironment even add

\footnotetext{
* Correspondence: syuankai@cicams.ac.cn

'Department of Medical Oncology, Beijing Key Laboratory of Clinical Study on Anticancer Molecular Targeted Drugs, National Cancer Center/National Clinical Research Center for Cancer/Cancer Hospital, Chinese Academy of Medical Sciences and Peking Union Medical College, No. 17 Panjiayuan Nanli, Chaoyang District, Beijing 100021, China

Full list of author information is available at the end of the article
}

another dimension into the complexity of tumor expansion [2]. Therefore, instead of single-target inhibitors currently used in cancer therapy, multi-targeted tyrosine kinase inhibitors (TKIs) that influence multiple key signal pathways in different type of tumor cells could overcome the limitations from single-target inhibition and be more efficient as promising therapeutic drugs due to their unique theoretical bases and anti-tumor mechanisms [3, 4].

Chiauranib is a novel orally active multi-target inhibitor that simultaneously inhibits three major pathways in tumorigenesis, including the angiogenesis-related kinases (VEGFR2, VEGFR1, VEGFR3, PDGFR $\alpha$, and c-Kit), 
mitosis-related kinase Aurora B, and chronic inflammation-related kinase CSF-1R. The compound shows very high selectivity and potency in the inhibition of these kinases with the IC50 at a single-digit nanomolar range, but with little activity on off-target non-receptor kinases, proteins, GPCR, and ion channels [5]. Target inhibition and effects on the corresponding biological pathways of chiauranib have been verified in vivo by preclinical studies. Also, chiauranib shows broad activity against human tumor xenograft models inoculated with human cancer cell lines derived from colon, lung, liver, or stomach cancers [5].

Based on the promising preclinical antitumor activity, this phase I study was designed to determine the safety and tolerability of chiauranib in patients with advanced solid tumor or lymphoma. The pharmacokinetic profile and preliminary antitumor activity were also evaluated as secondary objectives.

\section{Methods}

\section{Chemicals and reagents}

Chiauranib capsules were provided by Shenzhen Chipscreen Biosciences, Ltd. (Shenzhen, China). Methanol and acetonitrile were of HPLC grade (Fisher Scientific, Fair Lawn, NJ, USA). All other chemicals were of analytical reagent grade. Distilled, deionized water was produced by a Milli-Q Reagent Water System (Millipore, MA, USA).

\section{Eligibility criteria}

Eligible patients had histological or cytological confirmation of advanced solid tumor, or non-Hodgkin lymphoma (NHL), refractory to or without standard therapy options. Other inclusion criteria were as follows: body mass index (BMI) was between 18 and 28; age was between 18 and 65; Eastern Cooperative Oncology Group (ECOG) performance status was of 0 to 1 ; acceptable liver, renal, and bone marrow function including normal serum electrolytes were mandatory: hemoglobin $(\mathrm{Hb}) \geq$ $100 \mathrm{~g} / \mathrm{L}$ (no blood transfusion within 14 days); absolute neutrophil count $\left(\right.$ ANC) $\geq 1.5 \times 10^{9} / \mathrm{L}$; platelets $\geq 100 \times$ $10^{9} / \mathrm{L}$; serum creatinine $\leq 1.5 \times$ upper limit of normal (ULN); total bilirubin $\leq 1.5 \times$ ULN; alanine aminotransferase (ALT)/aspartate aminotransferase (AST) $\leq 1.5 \times$ $\mathrm{ULN}$; fasting triglyceride (TG) $\leq 3.0 \mathrm{mmol} / \mathrm{L}$; total cholesterol $\leq 7.75 \mathrm{mmol} / \mathrm{L}$; and International Normalized Ratio (INR) $<1.5$. Women of childbearing potential should be non-lactating patients and must agree to use effective contraceptive methods prior to study entry, during study participation, and up to 6 months following completion of therapy. A serum or urine pregnancy test within 7 days before enrollment must be negative. Men must agree to use effective contraceptive methods during study participation and up to 6 months following completion of therapy.

Major exclusion criteria included patients with life expectancy less than 3 months, or with uncontrolled or significant cardiovascular diseases, or with any anti-cancer treatment within 4 weeks prior to study entry. Other exclusion criteria were as follows: active bleeding, or current thrombotic disease, or using anticoagulants; history of deep venous thrombosis or pulmonary embolism; clinical significant gastrointestinal abnormality; brain metastasis with symptoms; and organ transplant recipients, proteinuria positive, congenital or acquired immunodeficiency, active infection, and mental disorders were ineligible.

The study (ClinicalTrials.gov ID: NCT02122809) was conducted according to good clinical practice guidelines and the Declaration of Helsinki. The Institutional Review Boards of the Cancer Hospital, Chinese Academy of Medical Sciences and Peking Union Medical College, approved the study, and written informed consent was obtained from all patients prior to performing study-related procedures.

\section{Study design}

This was an open-label, dose-escalation $(3+3$ design $)$ cohort study of single-agent chiauranib. The primary objective of the study was to determine dose-limiting toxicity (DLT), maximum tolerated dose (MTD), and safety profile. Secondary objectives were to characterize the pharmacokinetic profile and preliminary antitumor activity of chiauranib.

\section{Drug administration}

Chiauranib was supplied by Shenzhen Chipscreen Biosciences, Ltd. In order to evaluate the initial pharmacokinetics (PK) parameters after one dose, a 6-day-lead-in period was designed in the first treatment cycle, followed by the daily administration of chiauranib capsules. A treatment cycle was defined as 28 days. Chiauranib capsules were administered with fasting condition.

Based on the overall results from preclinical studies and the principle for human equivalent dose (HED) analysis, $25 \mathrm{mg} /$ day would be suggested as the starting dose for chiauranib in human. The executed starting dose in the study was $10 \mathrm{mg} /$ day, considering a safety factor of 2.5 , and only two patients were required to enroll in this group. The escalating doses were $20 \mathrm{mg}, 35 \mathrm{mg}, 50 \mathrm{mg}$, and $65 \mathrm{mg}$, determined by a modified Fibonacci sequence until the MTD was achieved.

\section{Dose-escalation procedures}

Patients were enrolled sequentially into dose escalation cohorts using a standard $3+3$ design, except that only two patients were enrolled at the starting dose. Intra-patient dose escalation was not permitted. 
If one of the first three patients at a dose level experienced DLT, up to three additional patients (total up to six patients) were enrolled at that dose level. If more than two patients at a dose level experienced DLT, dose escalation was halted, and the dose level was determined to have exceeded MTD. Three additional patients were then entered on the next lower dose level. MTD was defined as the highest dose with an observed incidence of DLT in no more than one of six patients.

\section{DLT definitions}

Toxicity was graded according to Common Terminology Criteria for Adverse Events (CTCAE) version 4.0. DLT was defined as any grade $\geq 2$ nephrotoxicity, or any grade $\geq 3$ non-hematologic toxicity, or grade $\geq 4$ hematologic toxicity occurring during the first cycle at least possibly related or of unknown relationship to chiauranib.

\section{Safety assessment and clinical evaluation}

Safety assessments included the reporting of all adverse events (AEs), changes in clinical laboratory parameters (hematology, serum chemistries, coagulation, urinalysis), vital sign measurements, 12-lead electrocardiogram (ECG) results, and physical examination findings. In addition, serial measurements of left ventricular ejection fraction, thyroid function, myocardial enzymes, troponin, amylase, and lipase were performed. Twenty-four-hour urine protein was implemented if clinically indicated.

Safety parameters were assessed at baseline and at regular intervals during the study. Adverse events (AEs) were classified/graded weekly according to the CTCAE version 4.0.

Tumor response was evaluated by the Response Evaluation Criteria in Solid Tumors (RECIST) version 1.1. Tumor response was assessed by computed tomography (CT) and/or magnetic resonance imaging (MRI) scans at screening and every 8 weeks ( 2 cycles) during the first 12 cycles. And the investigator could adjust the assessing intervals according to specific conditions of the patient thereafter, if patients continued to receive treatment with chiauranib for 13 or more cycles.

\section{Pharmacokinetic sampling and analysis}

In the initial PK evaluation after one dose, the eligible patients received a single oral dose of $10,20,35,50$, and $65 \mathrm{mg}$ chiauranib capsules under fasting condition. Three milliliters of venous blood samples was drawn into heparinized tubes before drug administration as well as at $1,2,4,6,8,12,24,48,72,96,120$, and $144 \mathrm{~h}$ post-dose. In the multiple dose study, patients continuously received from 10 to $65 \mathrm{mg}$ chiauranib capsules once daily at the first treatment cycle. Three milliliters of venous blood samples was drawn into heparinized tubes before administration as well as at $1,2,4,8,12$, and $24 \mathrm{~h}$ post the oral dose on day 28 . To evaluate the steady-state trough concentration of chiauranib, blood samples were also collected before dosing on days 8,15 , $22,25,26$, and 27 of the first cycle, and on each day 28 from the second cycle until the end. Samples were centrifuged immediately at $3600 \times g$ for $10 \mathrm{~min}$ at $4{ }^{\circ} \mathrm{C}$, and the plasma fractions were separated and stored at $-20^{\circ} \mathrm{C}$ until analysis.

The plasma concentration of chiauranib was determined by a validated LC-MS/MS method with lower limits of quantification (LOQ) at $1.00 \mathrm{ng} / \mathrm{mL}$. The intraand inter-day precisions and accuracies (relative standard deviation, \%RSD) were in the range of $1.75-6.33 \%$, and $-7.88-9.00 \%$, respectively. The extraction recovery (relative standard deviation, \%RSD) was $1.32-5.16 \%$.

\section{Statistical methods}

Descriptive statistics were used for baseline characteristics, safety assessments, pharmacokinetic variables (including $C_{\max }, T_{\max }, \mathrm{AUC}$, and $t_{1 / 2}$ ) and exploratory efficacy assessments, including tumor response and time to progression. Continuous variables were summarized with means, standard deviations (SD), medians, minimums, and maximums. Categorical variables were summarized by counts and by percentage of patients in corresponding categories. $P$ values $<0.05$ were considered statistically significant. Statistical analyses were performed using $\mathrm{SAS}^{\ominus}$ v9.4 (Cary, NC).

\section{Results}

\section{Patient characteristics}

Eighteen patients ( 6 female, 12 male) were enrolled from March 7, 2014, to December 30, 2015. The median age was 49.5 years (range 23-65). Eight patients (44\%) were of ECOG PS 0. All patients had confirmed progressive metastatic diseases at study entry with a median of 3.5 prior regimens of systemic therapies (range 1-9). The most common primary malignancies were colorectal cancer (7 cases, 38.9\%), non-small cell lung cancer (NSCLC) (5 cases, 27.8\%), and others (6 cases) with multiple primary cancers. Baseline patient and tumor characteristics are detailed in Table 1.

\section{Dose exposure and modifications}

A total of 87 treatment cycles were administered to 18 patients at 5 different dose levels during the study. The duration of treatment was consistent across the dose levels, with a median of 3 cycles being administered at all dose levels (range 1-23). Ten patients (55.6\%) were continued for treatment after 2 cycles, and all of them completed at least 3 cycles with the longest duration of treatment for 23 cycles. At the time of data analysis, all 18 patients stopped therapy for following reasons: 14 (77.8\%) due to disease progression, 1 (5.6\%) due to DLT 
Table 1 Baseline demographics and patient characteristics

\begin{tabular}{ll}
\hline Characteristic & Total $(N=18)$ \\
\hline Sex, $n$ (\%) & $12(66.7)$ \\
Male & $6(33.3)$ \\
Female & 49.1 \\
Age (mean), years & $49.5(23-65)$ \\
Median (range) & \\
ECOG performance status, $n$ (\%) & $8(44.4)$ \\
0 & $10(55.6)$ \\
1 & $1(6)$ \\
TNM stage, $n$ (\%) & $17(94)$ \\
IIIA & \\
IV & $3.5(1-9)$ \\
Previous regimens of systemic therapies & \\
Median (range) & \\
Tumor type, $n$ (\%) & $5(27.8)$ \\
NSCLC & $7(38.9)$ \\
Colorectal cancer & $1(5.6)$ \\
Ovarian cancer & $1(5.6)$ \\
Gastric cancer & $1(5.6)$ \\
Thyroid cancer & $1(5.6)$ \\
DLBCL & $1(5.6)$ \\
Fibrosarcoma & $1(5.6)$ \\
\hline Renal cancer & \\
$D L B C L$ diffuse large B cell lymphoma & \\
&
\end{tabular}

(treatment-related grade 3 non-hematologic toxicity) at dose $65 \mathrm{mg}$ during cycle 1, 1 (5.6\%) due to withdrawal of consent, and $2(11.1 \%)$ due to investigator's discretion. For the investigator's discretion of treatment termination, 1 patient was due to poor general condition in her late-stage disease. The other patient with diffuse large B cell lymphoma was treated by the study drug at $10 \mathrm{mg}$ for 23 cycles with long-lasting stabilized disease lesions showing no metabolism activity by Positron emission tomography-computer tomography (PET-CT) assessment.

Of the 18 enrolled patients, none underwent dose modifications or reductions, but treatment delay was implemented on 2 (11.1\%) patients because of adverse events: 1 patient in the $10 \mathrm{mg}$ cohort omitted doses for 5 days due to grade 1 nasopharyngitis and 1 patient treated at dose level $50 \mathrm{mg}$ omitted treatment for 10 days due to grade 1 elevation of AST and ALT. These adverse events resolved fully following brief drug holidays. The elevation of AST and ALT was considered by the investigator to be possibly related to chiauranib, and the nasopharyngitis was considered to be unlikely related. All patients who received study treatment were evaluable for safety, pharmacokinetics, and efficacy.
Dose escalation and recommended phase II dose

All the 18 patients were evaluable for the determination of MTD during the first 4 weeks of treatment only (1 cycle). Two patients were enrolled in the initial dosing cohort of $10 \mathrm{mg}$ daily. Dose levels were escalated in four additional cohorts: $20 \mathrm{mg}$ daily $(n=6), 35 \mathrm{mg}$ daily $(n=$ $3), 50 \mathrm{mg}$ daily $(n=3)$, and $65 \mathrm{mg}$ daily $(n=4)$. Two of the 4 patients at the highest dose level experienced protocol-defined DLTs of grade 3 hypertension during cycle 1 , prompting a decision not to escalate further. Hypertension only resulted in treatment discontinuation for 1 patient, while the other patient who underwent DLT was assessed by the investigator to continue treatment due to potential and promising benefit from chiauranib. Except for hypertension, no other DLT was reported during the study (All other events were either not considered to be related to treatment by the investigator or did not conform to the DLT definition).

Based on the safety data, $50 \mathrm{mg}$ chiauranib administered orally once daily was considered to be both the MTD and the RP2D.

\section{Safety and adverse event profile}

Chiauranib was generally well tolerated, and the majority of AEs were mild to moderate in severity. No treatment-related deaths occurred during the study. All 18 patients experienced at least one $\mathrm{AE}$, and 17 patients (94.4\%) reported at least one treatment-related AE. The most common treatment-related AEs, irrespective of grade, were fatigue (61.1\%), proteinuria (44.4\%), hematuria (38.9\%), hypothyroidism (38.9\%), hypertriglyceridemia (33.3\%), and hypertension (33.3\%). There were no obvious abnormal laboratory parameters for renal function observed, such as serum urea nitrogen and creatinine. Table 2 summarized the most common treatment-related AEs occurring in at least $10 \%$ of patients. All these treatment-related AEs were of grade 1 or 2 severity with the exception of five cases, which were grade 3 hypertension (in two patients), neutropenia (in two patients), and hyperglycemia (in one patient). No treatment-related $\mathrm{AE}$ exceeded grade 3 was observed.

\section{Serious adverse event}

A total of one serious adverse event (SAE) was reported. One patient with advanced NSCLC treated at the 20-mg dose level experienced AEs comprising grade 2 cough, fatigue, and anorexia during the first cycle, and the investigator made a decision that the patient withdraw from the study due to her poor condition. This patient died from deteriorating pulmonary infection 15 days after treatment termination of the study drug. Although this death was assessed as unlikely related to the study 
Table 2 Treatment-related adverse events: worst grade per patient in all cycles in at least 10\% of all patients, $n$ (\%)

\begin{tabular}{|c|c|c|c|c|c|c|}
\hline \multirow[t]{2}{*}{ Adverse event } & \multicolumn{5}{|l|}{ Grade $^{a}$} & \multirow[t]{2}{*}{ Total } \\
\hline & $\overline{1}$ & 2 & 3 & 4 & 5 & \\
\hline \multicolumn{7}{|l|}{ Blood and lymphatic system disorders } \\
\hline Hypertension & $3(16.7)$ & $1(5.6)$ & $2(11.1)$ & 0 & 0 & $6(33.3)$ \\
\hline Vaginal hemorrhage & $2(11.1)$ & 0 & 0 & 0 & 0 & $2(11.1)$ \\
\hline \multicolumn{7}{|l|}{ Endocrine disorders } \\
\hline Hypothyroidism & $7(38.9)$ & 0 & 0 & 0 & 0 & $7(38.9)$ \\
\hline \multicolumn{7}{|l|}{ Metabolism and nutrition disorders } \\
\hline Hypertriglyceridemia & $5(27.8)$ & $1(5.6)$ & 0 & 0 & 0 & $6(33.3)$ \\
\hline Decreased appetite & 0 & $2(11.1)$ & 0 & 0 & 0 & $2(11.1)$ \\
\hline \multicolumn{7}{|l|}{ Respiratory, thoracic, and mediastinal disorders } \\
\hline Dysphonia & $2(11.1)$ & 0 & 0 & 0 & 0 & $2(11.1)$ \\
\hline \multicolumn{7}{|l|}{ Gastrointestinal disorders } \\
\hline Nausea & $4(22.2)$ & 0 & 0 & 0 & 0 & $4(22.2)$ \\
\hline Abdominal pain & $2(11.1)$ & 0 & 0 & 0 & 0 & $2(11.1)$ \\
\hline Diarrhea & $2(11.1)$ & $1(5.6)$ & 0 & 0 & 0 & $3(16.7)$ \\
\hline Mouth ulceration & $1(5.6)$ & $1(5.6)$ & 0 & 0 & 0 & $2(11.1)$ \\
\hline \multicolumn{7}{|l|}{ Renal and urinary disorders } \\
\hline Proteinuria & $8(44.4)$ & 0 & 0 & 0 & 0 & $8(44.4)$ \\
\hline Hematuria & $7(38.9)$ & 0 & 0 & 0 & 0 & $7(38.9)$ \\
\hline \multicolumn{7}{|l|}{ Skin and subcutaneous tissue disorders } \\
\hline Rash & $1(5.6)$ & $1(5.6)$ & 0 & 0 & 0 & $2(11.1)$ \\
\hline Palmar-plantar erythrodysesthesia syndrome & $1(5.6)$ & $1(5.6)$ & 0 & 0 & 0 & $2(11.1)$ \\
\hline \multicolumn{7}{|l|}{ General disorders and administration site conditions } \\
\hline Fatigue & $7(38.9)$ & $4(22.2)$ & 0 & 0 & 0 & $11(61.1)$ \\
\hline \multicolumn{7}{|l|}{ Lab examinations } \\
\hline Leucopenia & $2(11.1)$ & $1(5.6)$ & 0 & 0 & 0 & $3(16.7)$ \\
\hline ALT increased & $2(11.1)$ & 0 & 0 & 0 & 0 & $2(11.1)$ \\
\hline Amylase increased & $3(16.7)$ & $1(5.6)$ & 0 & 0 & 0 & $4(22.2)$ \\
\hline Conjugated bilirubin increased & $5(27.8)$ & 0 & 0 & 0 & 0 & $5(27.8)$ \\
\hline Neutropenia & $2(11.1)$ & 0 & $2(11.1)$ & 0 & 0 & $4(22.2)$ \\
\hline AST increased & $3(16.7)$ & 0 & 0 & 0 & 0 & $3(16.7)$ \\
\hline Hyperbilirubinemia & $2(11.1)$ & 0 & 0 & 0 & 0 & $2(11.1)$ \\
\hline Unconjugated bilirubin increased & $2(11.1)$ & 0 & 0 & 0 & 0 & $2(11.1)$ \\
\hline Blood urea increased & $2(11.1)$ & 0 & 0 & 0 & 0 & $2(11.1)$ \\
\hline Thrombocytopenia & $2(11.1)$ & 0 & 0 & 0 & 0 & $2(11.1)$ \\
\hline
\end{tabular}

Patients with multiple events in the same category are counted only once in that category; patients with events in more than one category are counted once in each of those categories

ALT alanine amino-transferase, AST aspartate amino-transferase

${ }^{\mathrm{a}}$ Graded per National Cancer Institute Common Terminology Criteria for Adverse Events, version 4.0

drug, six patients were enrolled in the $20 \mathrm{mg}$ cohort in this study.

\section{Pharmacokinetic studies}

Over the dose escalation from 10 to $65 \mathrm{mg}$ in patients, chiauranib plasma exposures showed a dose proportional and good linear pharmacokinetic profile in both single and multiple dose studies (Fig. 1a-c). After the first dose administration in run-in period, chiauranib exhibited a rapid absorption with a median peak time of 2 $\mathrm{h}$ (range from 1 to $4 \mathrm{~h}$ ), and a relatively slow elimination with the terminal half-life $\left(t_{1 / 2}\right)$ of $25.6 \pm 6.73 \mathrm{~h}$. The accumulative exposure of chiauranib such as mean AUC and $C_{\max }$ on day 28 in the first treatment cycle was dose 


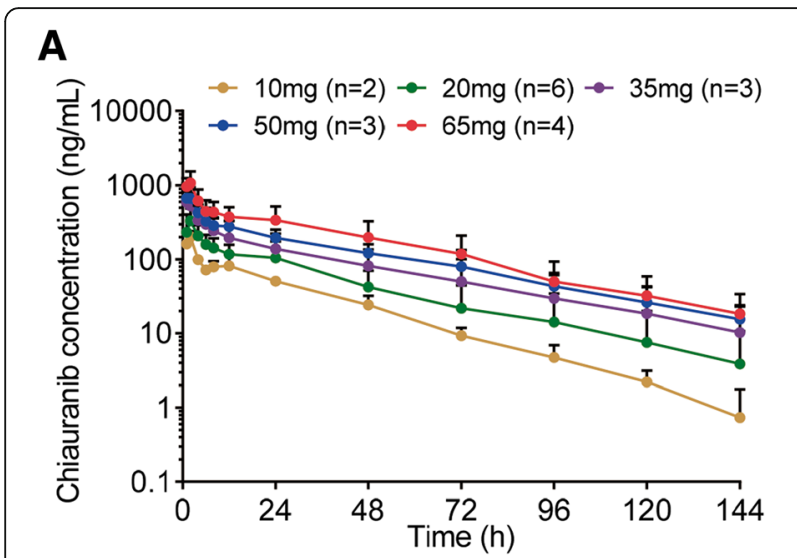

B

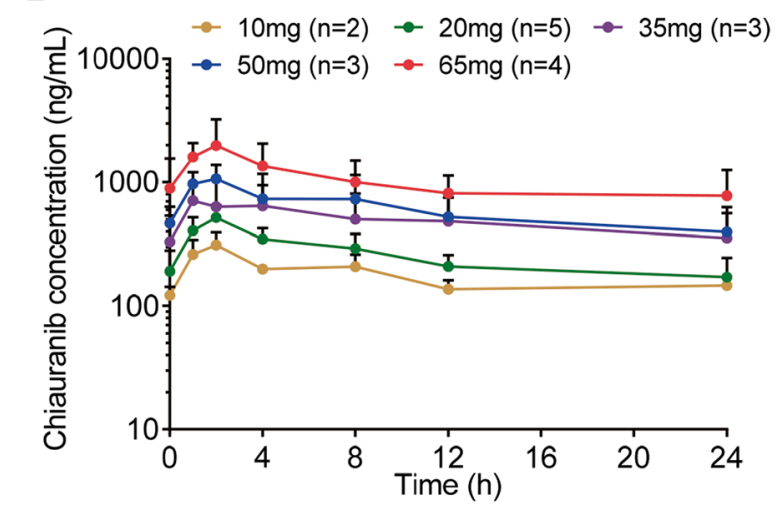

C

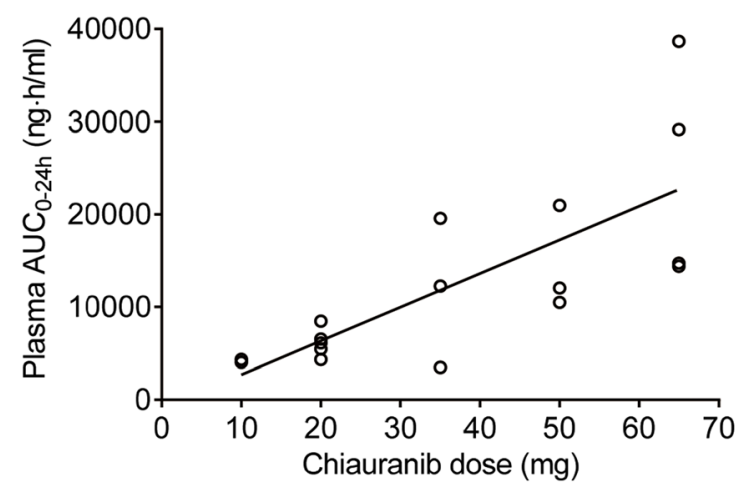

Fig. 1 Pharmacokinetic analyses of chiauranib. a The mean plasma concentration-time curves of chiauranib in patients at a single-oral dose of 10, 20, 35, 50, and $65 \mathrm{mg}$ chiauranib. The mean plasma concentration-time curves (b) and AUC-dose linear regression (c) of chiauranib on day 28 in the first treatment cycle at a multiple oral dose of 10, 20, 35, 50, and 65 mg chiauranib

proportional and approximately increased by twofold compared to those in the single dose study. Notably, the continuous dosing of chiauranib did not result in the saturable absorption even at the clinical DLT of $65 \mathrm{mg}$. The plasma exposure parameters of chiauranib for both single and multiple dose are listed in Table 3.
The plasma exposure of chiauranib reached the steady state from day 8 upon continuous once daily administration. The trough plasma concentration of chiauranib was consistently stable from the first treatment cycle, and increased by twofold compared to that after the first administration of chiauranib (Fig. 2).

\section{Anti-tumor activity}

Tumor response was assessed during the study. Among the 18 patients enrolled, 15 had baseline measurable diseases evaluable for tumor response by RECIST 1.1. No partial or complete response was observed; 12 patients (66.7\%) had stable disease (SD), and 6 patients (33.3\%) had progressive disease (PD) as best response to therapy based on the investigator's assessment. Figure 3a represents the changes in the sum of the longest diameters of target lesions observed at the time of best response.

Patients who experienced clinical benefit for greater than 8 weeks included two patients with colorectal cancer (one patient each in the 20 and $50 \mathrm{mg}$ cohorts), two patients with NSCLC (one patient each in the 20 and 35 $\mathrm{mg}$ cohorts), one patient with ovarian cancer $(65 \mathrm{mg}$ cohort), and one patient with DLBCL (10 mg cohort). Typically, one patient with NSCLC (poorly differentiated adenocarcinoma) in the dose of $20 \mathrm{mg}$ cohort had showed diminished pericardial effusion and tumor shrinkage with a cavity in the chest $\mathrm{CT}$ at the time of 4 weeks treatment (Fig. 3b, c).

\section{Discussion}

With the advanced molecular biology and understanding of tumorigenesis, an increasing number of kinase-mediated signaling pathways have been identified as potential therapeutic targets for the treatment of hematologic malignancies and solid tumors [6]. Chiauranib is a novel orally active multi-target inhibitor that simultaneously inhibits the angiogenesis-related kinases (VEGFR2, VEGFR1, VEGFR3, PDGFR $\alpha$, and c-Kit), mitosis-related kinase Aurora B, and chronic inflammation-related kinase CSF-1R. The potential anti-tumor activities of chiauranib have been previously demonstrated both in vitro and in vivo xenograft tumor models [5].

The current study was undertaken to determine the safety, DLT, and RP2D of chiauranib. In general, chiauranib was well tolerated with mild to moderate toxicity profile, and the most frequently reported treatmentrelated AEs were fatigue, proteinuria, hematuria, hypothyroidism, hypertriglyceridemia, hypertension, bilirubin increased, and neutropenia, as expected from the mechanism of action of the drug. For example, proteinuria and hypothyroidism have also been reported with other VEGFR TKIs, probably due to a loss of endothelial fenestrations and podocytes [7] and thyroid atrophy from reduction of vessel density [8]. 
Table 3 The pharmacokinetic exposure parameters of plasma chiauranib after single and multiple dose of 10, 20, 35, 50, and 65 mg chiauranib capsules in patients with advanced solid tumor

\begin{tabular}{|c|c|c|c|c|c|c|c|c|}
\hline & & $T_{\max }(\mathrm{h})$ & $\mathrm{AUC}_{0-24 \mathrm{~h}}(\mathrm{ng} \mathrm{h} / \mathrm{mL})$ & $\mathrm{AUC}_{0-144 \mathrm{~h}}(\mathrm{ng} \mathrm{h} / \mathrm{mL})$ & $C_{\max }(\mathrm{ng} / \mathrm{mL})$ & DF & $C_{\text {trough }}(\mathrm{ng} / \mathrm{mL})$ & $C_{a v}(\mathrm{ng} / \mathrm{mL})$ \\
\hline \multirow[t]{5}{*}{ Day1 } & $10 \mathrm{mg}(n=2)$ & 2.0 & 1998 & 3576 & 201 & 1 & / & 1 \\
\hline & $20 \mathrm{mg}(n=6)$ & $2.0(1-4)$ & 3454 (1275) & 6815 (3902) & 354 (158) & / & / & / \\
\hline & $35 \mathrm{mg}(n=3)$ & $1.0(1-8)$ & 5762 (3853) & 11859 (8656) & $608(437)$ & / & / & / \\
\hline & $50 \mathrm{mg}(n=3)$ & $1.0(1-2)$ & 7417 (1380) & $16429(5251)$ & 756 (87) & / & / & / \\
\hline & $65 \mathrm{mg}(n=4)$ & $1.5(1-2)$ & 11015 (3572) & $24823(12581)$ & 1275 (358) & / & / & / \\
\hline \multirow[t]{5}{*}{ Day28 } & $10 \mathrm{mg}(n=2)$ & 2.0 & 4185 & / & 312 & $1.1(0.4)$ & $122(21)$ & $174(9)$ \\
\hline & $20 \mathrm{mg}(n=6)$ & 2.0 & $6175(1526)$ & / & 517 (134) & $1.3(0.6)$ & $191(89)$ & 257 (64) \\
\hline & $35 \mathrm{mg}(n=3)$ & $4(1-4)$ & 11761 (8066) & / & 773 (532) & $0.8(0.4)$ & $330(208)$ & 490 (336) \\
\hline & $50 \mathrm{mg}(n=3)$ & $2(1-2)$ & $14504(5650)$ & / & $1118(254)$ & $1.1(0.3)$ & $467(166)$ & $604(235)$ \\
\hline & $65 \mathrm{mg}(n=4)$ & $2(1-2)$ & 24230 (11819) & / & 2238 (1012) & $1.5(0.8)$ & $890(662)$ & $1010(492)$ \\
\hline
\end{tabular}

$A U C_{0-24 \mathrm{~h}}$ area under the concentration-time curve from 0 to $24 \mathrm{~h}, A \cup C_{0-144 \mathrm{~h}}$ area under the concentration-time curve from 0 to $144 \mathrm{~h}, C_{\max }$ maximum plasma concentration, $T_{\max }$ time to reach maximum plasma concentration

Neutropenia is another common toxicity in our study, which could be related to the inhibition of Aurora B [9] and CSF-1R kinases [10].

During dose escalation, two cases of grade 3 hypertension were observed as DLTs at $65 \mathrm{mg}$ cohort. Hypertension is often a dose-limiting toxicity for VEGFR inhibition, which may be a mechanism-dependent, "on-target" toxicity of the VEGF signaling pathway rather than non-specific effects on unrelated signaling pathways $[11,12]$. Other DLTs of VEGFR inhibitors including fatigue, skin rash, palmar-plantar erythrodysesthesia syndrome, and diarrhea were observed in early clinical studies [7, 13, 14]. DLTs in phase I trials of Aurora kinase inhibitors were primarily hematological events, particularly neutropenia [15-18], and DLTs for CSF-1R inhibitor included INR increase, lymphopenia, AST increase, anemia, neutropenia, and syncope [10]. As for multi-target inhibitors, Ilorasertib (ABT-348)

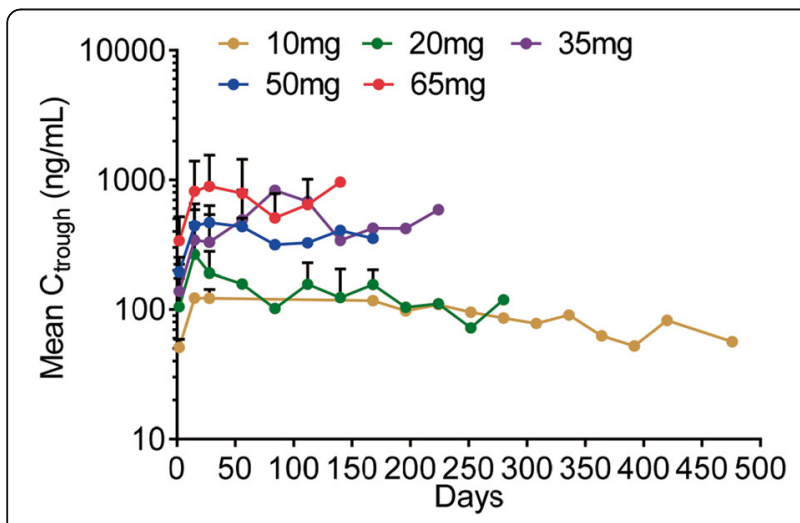

Fig. 2 Steady-state mean trough concentration $\left(C_{\text {trough }}\right)$ of chiauranib. Mean $C_{\text {trough }}$ after a continuous oral dose of 10, 20, 35, 50, and $65 \mathrm{mg}$ chiauranib capsules once daily in patients throughout the treatment cycles were shown targeting Aurora kinase A/B, VEGFR/PDGFR and Src family, and ENMD-2076 targeting Aurora kinase A, VEGFR2/FGFR/Flt-3/c-Kit, were found to cause more DLTs, such as pancreatic disease and hypertension, fatigue, cholecystitis, and QTc prolonged [19, 20]. Comparing to these single- or multi-target inhibitors above mentioned, chiauranib showed less DLTs and all the grade 3 AEs such as hypertension, hyperglycemia, and neutropenia were highly manageable. On the basis of these results, the PR2D of chiauranib was $50 \mathrm{mg}$ for once-daily administration as monotherapy.

Chiauranib was well absorbed and showed favorable pharmacokinetic parameters. Chiauranib performed typical linear pharmacokinetic profile in both single and multiple dose studies, in which the main parameters $C_{\max }$ and AUC showed linear relation to the dosages. And it did not present any evidence of the saturation of absorption even at the dose of DLT. Compared to the drug exposure after the single-dose administration, the accumulative exposure of chiauranib in the steady state was approximately increased by twofold. Also, the steady state $C_{\text {trough }}$ was consistent and relatively stable in patients from the first treatment cycle. Overall, the results of single/multiple doses study indicate that chiauranib has well pharmacokinetic characters with tolerance and safety, which provide useful information for the further clinical trial.

In the present phase 1 trial, 12 patients (66.7\%) achieved SD as the best response. No complete or partial responses were observed. All of these cases who achieved SD greater than 8 weeks included colorectal cancer, NSCLC, ovarian cancer, and DLBCL. Among them, one patient with ovarian cancer demonstrated a marginal partial response with $22.2 \%$ change in the sum of the longest diameters of target lesions observed at the time of best response. Furthermore, one patient with 


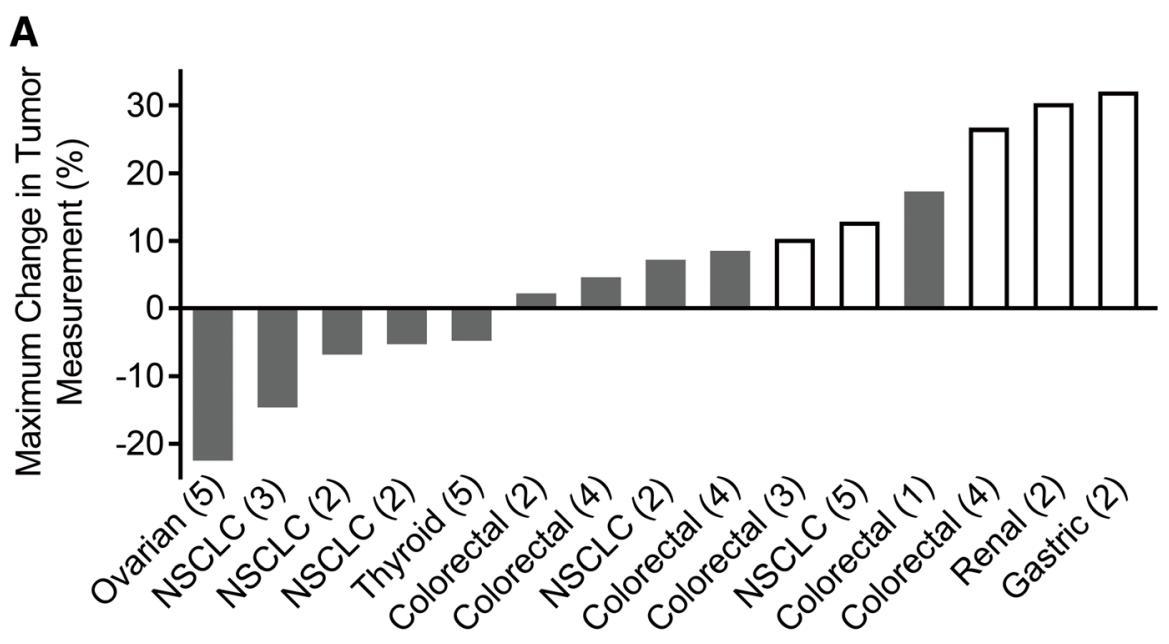

B

\section{C}
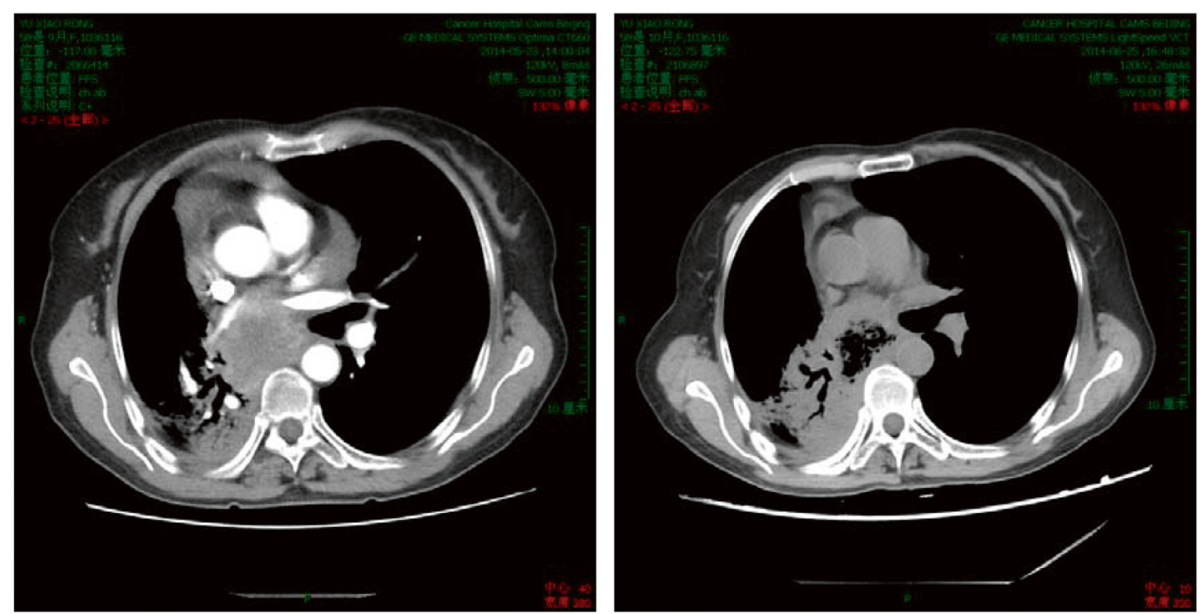

Fig. 3 Waterfall plot of changes in the target lesions with a representative case of clinical response. a Chiauranib dose cohorts are shown in parentheses as $1=10 \mathrm{mg}, 2=20 \mathrm{mg}, 3=35 \mathrm{mg}, 4=50 \mathrm{mg}$, and $5=65 \mathrm{mg}$. Open bars represent patients who had progressive disease, and the filled bars represent patients who achieved stable disease as their best responses. Not shown, $n=3$, due to unavailable tumor measurements. Coronal chest $C T$ at baseline (b) and after 4 weeks chiauranib treatment (c) from a 59-year-old woman with poorly differentiated adenocarcinoma of the lung, who has been received surgery, adjuvant chemotherapy, chemo-radiation, and two salvage chemotherapies

DLBCL in the $10 \mathrm{mg}$ cohort experienced a confirmed SD lasting for 596 days.

Notably, in this heavily pretreated population, six patients $(6 / 18,33.3 \%)$ with a variety of solid tumors, having received prior anti-angiogenic agents, also showed prolonged clinical benefits. While five patients achieved SD, four among them remained on chiauranib for at least 16 weeks. This may indicate a better clinical efficacy of chiauranib compared with other anti-angiogenic agents, likely because of the concomitant inhibition of Aurora kinase and CSF-1/CSF-1R signaling pathways. Possibly, prior exposure to antiangiogenic therapy did not preclude clinical benefit of chiauranib. Although a correlation between the development of hypertension and clinical benefit has been reported for a number of antiangiogenic agents, including bevacizumab, sunitinib, and sorafenib [21-24], we did not observe this association with chiauranib.

In summary, this first-in-human study demonstrated that the novel, angiogenic, Aurora, and CSF1-R kinase inhibitor chiauranib is well tolerated and can be safely administered to cancer patients with a PR2D of $50 \mathrm{mg}$ QD once daily. Favorable PK properties were confirmed, and signs of clinical activity were elicited. Evaluation of chiauranib as monotherapy in four phase Ib/II clinical trials of relapsed/refractory ovarian cancer, NHL, hepatic cell carcinoma, and small cell lung cancer patients after standard systemic therapy (ClinicalTrials.gov NCT03166891, 03074825, 03245190, and 03216343) are currently undergoing. 


\section{Abbreviations}

AE: Adverse event; $\mathrm{Cl}$ : Confidence interval; $C_{\text {max }}$ : Maximum plasma concentration; CTCAE: National Cancer Institute Common Terminology Criteria for Adverse Events; CYP: Cytochrome; DLBCL: Diffuse large B cell lymphoma; DLT: Dose-limiting toxicity; ECOG: Eastern Cooperative Oncology Group; MTD: Maximum tolerated dose; NA: Not applicable; NSCLC: Non-smal cell lung cancer; PK: Pharmacokinetics; RECIST: Response Evaluation Criteria in Solid Tumors; RP2D: Recommended phase II dose; SAE: Serious adverse event; SD: Stable disease; TKI: Tyrosine kinase inhibitor; $T_{\max }$ : Time to reach maximum plasma concentration; WHO: World Health Organization

\section{Acknowledgements}

The authors would like to thank the patients, study investigators, coordinators, and study site personnel who participated in this study. The authors also thank Drs. LJ Xin and SY Jiang for critical reading of the manuscript. We thank Shenzhen Chipscreen Biosciences, Ltd. for sponsoring this study and XBL-China for detecting and analyzing the PK samples.

\section{Funding}

The study was supported by grants from the National Science and Technology Major Projects (2011ZX09102-001-09, 2013ZX09401301, and 2018ZX09301020-

003), the National Major Project for New Drug Innovation (2012ZX09303012-

001), the Significant Project in Biotech Field from Shenzhen City (GJHS20120820153947138), and the Collaborative Innovation Project from Shenzhen Science and Technology Innovation Committee (GJHS20160831155623609).

\section{Availability of data and materials}

The dataset used and/or analyzed during the current study are available from the corresponding author on reasonable request.

\section{Authors' contributions}

YKShi was the principal investigator of this clinical trial and contributed to study design and conception. YKShi, YKSun, LY, XZH, and YTL were involved in patient recruitment, treatment, data acquisition, and analysis, and JWZ was involved in the analysis of PK results. All authors were involved in writing the paper and had full access to the data in this study. All authors had reviewed and approved the final version of this manuscript. The corresponding author had final responsibility for the decision to submit the manuscript for publication.

\section{Ethics approval and consent to participate}

All patients provided written informed consent. The protocol was approved by the institutional review boards of the Cancer Hospital, Chinese Academy of Medical Sciences and Peking Union Medical College.

\section{Consent for publication}

The consent to publish has been obtained from the participants to report individual patient data.

\section{Competing interests}

ZQ Ning and JW Zhang are employees of Shenzhen Chipscreen Biosciences, Ltd. The other authors declared that they have no competing interest.

\section{Publisher's Note}

Springer Nature remains neutral with regard to jurisdictional claims in published maps and institutional affiliations.

\section{Author details}

'Department of Medical Oncology, Beijing Key Laboratory of Clinical Study on Anticancer Molecular Targeted Drugs, National Cancer Center/National Clinical Research Center for Cancer/Cancer Hospital, Chinese Academy of Medical Sciences and Peking Union Medical College, No. 17 Panjiayuan Nanli, Chaoyang District, Beijing 100021, China. ${ }^{2}$ Shenzhen Chipscreen Biosciences Ltd., BIO-Incubator, Suit 2-601, Shenzhen Hi-Tech Industrial Park, Shenzhen 518057, Guangdong, China.
Received: 25 September 2018 Accepted: 27 December 2018

Published online: 14 January 2019

\section{References}

1. Hanahan D, Weinberg RA. Hallmarks of cancer: the next generation. Cell. 2011;144:646-74.

2. Junttila MR, de Sauvage FJ. Influence of tumour micro-environment heterogeneity on therapeutic response. Nature. 2013;501:346-54.

3. Zhang J, Yang PL, Gray NS. Targeting cancer with small molecule kinase inhibitors. Nat Rev Cancer. 2009;9:28-39.

4. Tan HY, Wang N, Lam W, Guo W, Feng Y, Cheng YC. Targeting tumour microenvironment by tyrosine kinase inhibitor. Mol Cancer. 2018;17:43.

5. Zhou Y, Shan S, Li ZB, Xin LJ, Pan DS, Yang QJ, Liu YP, Yue XP, Liu XR, Gao JZ, et al. CS2164, a novel multi-target inhibitor against tumor angiogenesis, mitosis and chronic inflammation with anti-tumor potency. Cancer Sci. 2017;108:469-77.

6. Gross S, Rahal R, Stransky N, Lengauer C, Hoeflich KP. Targeting cancer with kinase inhibitors. J Clin Invest. 2015;125:1780-9.

7. Falchook GS, Wheler JJ, Naing A, Piha-Paul SA, Fu S, Tsimberidou AM, Hong DS, Janku F, Zinner R, Jiang $Y$, et al. Dual antiangiogenic inhibition: a phase dose escalation and expansion trial targeting VEGF-A and VEGFR in patients with advanced solid tumors. Invest New Drugs. 2015;33:215-24.

8. Verheul HM, Pinedo HM. Possible molecular mechanisms involved in the toxicity of angiogenesis inhibition. Nat Rev Cancer. 2007;7:475-85.

9. Collins GP, Eyre TA, Linton KM, Radford J, Vallance GD, Soilleux E, Hatton C. A phase II trial of AZD1152 in relapsed/refractory diffuse large B-cell lymphoma. Br J Haematol. 2015;170:886-90.

10. Tap WD, Wainberg ZA, Anthony SP, Ibrahim PN, Zhang C, Healey JH, Chmielowski B, Staddon AP, Cohn AL, Shapiro Gl, et al. Structure-guided blockade of CSF1R kinase in tenosynovial giant-cell tumor. N Engl J Med. 2015:373:428-37.

11. Robinson ES, Khankin EV, Karumanchi SA, Humphreys BD. Hypertension induced by vascular endothelial growth factor signaling pathway inhibition: mechanisms and potential use as a biomarker. Semin Nephrol. 2010;30:591-601.

12. Small HY, Montezano AC, Rios FJ, Savoia C, Touyz RM. Hypertension due to antiangiogenic cancer therapy with vascular endothelial growth factor inhibitors: understanding and managing a new syndrome. Can J Cardiol. 2014;30:534-43.

13. Strumberg D, Richly H, Hilger RA, Schleucher N, Korfee S, Tewes M, Faghih M, Brendel E, Voliotis D, Haase CG, et al. Phase I clinical and pharmacokinetic study of the Novel Raf kinase and vascular endothelial growth factor receptor inhibitor BAY 43-9006 in patients with advanced refractory solid tumors. J Clin Oncol. 2005;23:965-72.

14. Drevs J, Siegert P, Medinger M, Mross K, Strecker R, Zirrgiebel U, Harder J, Blum H, Robertson J, Jurgensmeier JM, et al. Phase I clinical study of AZD2171, an oral vascular endothelial growth factor signaling inhibitor, in patients with advanced solid tumors. J Clin Oncol. 2007:25:3045-54.

15. Falchook G, Kurzrock R, Gouw L, Hong D, McGregor KA, Zhou X, Shi H, Fingert $\mathrm{H}$, Sharma S. Investigational Aurora A kinase inhibitor alisertib (MLN8237) as an enteric-coated tablet formulation in non-hematologic malignancies: phase 1 dose-escalation study. Invest New Drugs. 2014;32: 1181-7.

16. Kantarjian HM, Sekeres MA, Ribrag $V$, Rousselot $P$, Garcia-Manero $G$, Jabbour EJ, Owen K, Stockman PK, Oliver SD. Phase I study assessing the safety and tolerability of barasertib (AZD1152) with low-dose cytosine arabinoside in elderly patients with AML. Clin Lymphoma Myeloma Leuk. 2013;13:559-67.

17. Dittrich C, Fridrik MA, Koenigsberg R, Lee C, Goeldner RG, Hilbert J, Greil R. A phase 1 dose escalation study of Bl 831266, an inhibitor of Aurora kinase $B$, in patients with advanced solid tumors. Invest New Drugs. 2015;33:409-22.

18. Mross K, Richly H, Frost A, Scharr D, Nokay B, Graeser R, Lee C, Hilbert J, Goeldner RG, Fietz O, Scheulen ME. A phase I study of BI 811283, an Aurora B kinase inhibitor, in patients with advanced solid tumors. Cancer Chemother Pharmacol. 2016;78:405-17.

19. Garcia-Manero G, Tibes R, Kadia T, Kantarjian H, Arellano M, Knight EA Xiong $H$, Qin Q, Munasinghe W, Roberts-Rapp L, et al. Phase 1 dose escalation trial of ilorasertib, a dual Aurora/NEGF receptor kinase inhibitor, in patients with hematologic malignancies. Invest New Drugs. 2015;33:870-80.

20. Diamond JR, Bastos BR, Hansen RJ, Gustafson DL, Eckhardt SG, Kwak EL, Pandya SS, Fletcher GC, Pitts TM, Kulikowski GN, et al. Phase I safety, 
pharmacokinetic, and pharmacodynamic study of ENMD-2076, a novel angiogenic and Aurora kinase inhibitor, in patients with advanced solid tumors. Clin Cancer Res. 2011;17:849-60.

21. Rixe $\mathrm{O}$, Billemont $\mathrm{B}$, Izzedine $\mathrm{H}$. Hypertension as a predictive factor of Sunitinib activity. Ann Oncol. 2007;18:1117.

22. Ryanne Wu R, Lindenberg PA, Slack R, Noone AM, Marshall JL, He AR.

Evaluation of hypertension as a marker of bevacizumab efficacy. J Gastrointest Cancer. 2009;40:101-8.

23. Estfan B, Byrne M, Kim R. Sorafenib in advanced hepatocellular carcinoma: hypertension as a potential surrogate marker for efficacy. Am J Clin Oncol. 2013:36:319-24.

24. Zhong J, Ali AN, Voloschin AD, Liu Y, Curran WJ Jr, Crocker IR, Shu HK. Bevacizumab-induced hypertension is a predictive marker for improved outcomes in patients with recurrent glioblastoma treated with bevacizumab. Cancer. 2015:121:1456-62.

Ready to submit your research? Choose BMC and benefit from:

- fast, convenient online submission

- thorough peer review by experienced researchers in your field

- rapid publication on acceptance

- support for research data, including large and complex data types

- gold Open Access which fosters wider collaboration and increased citations

- maximum visibility for your research: over $100 \mathrm{M}$ website views per year

At BMC, research is always in progress.

Learn more biomedcentral.com/submissions 